\title{
2020 Chinese Society of Clinical Oncology Breast Cancer guideline updates
}

\author{
Bo Shen $^{1,2}$, Kun Wang ${ }^{1}$, Zefei Jiang ${ }^{3}$ \\ ${ }^{1}$ Department of Breast Cancer, Cancer Center, Guangdong Provincial People's Hospital and Guangdong Academy of Medical Sciences, Guangzhou, \\ China; ${ }^{2}$ Shantou University Medical College, Shantou, China; ${ }^{3}$ Department of Breast Oncology, The Fifth Medical Center of Chinese PLA General \\ Hospital, Beijing, China \\ Correspondence to: Kun Wang. Department of Breast Cancer, Cancer Center, Guangdong Provincial People’s Hospital and Guangdong Academy \\ of Medical Sciences, Guangzhou, China. Email: gzwangkun@126.com; Zefei Jiang. Department of Breast Oncology, The Fifth Medical Center of \\ Chinese PLA General Hospital, No. 8 Dong Street, Fengtai District, Beijing, China. Email: jiangzefei@csco.org.cn.
}

Received: 09 August 2020; Accepted: 10 September 2020; Published: 31 October 2020.

doi: $10.21037 /$ tbcr-20-50

View this article at: http://dx.doi.org/10.21037/tbcr-20-50

\section{Introduction}

Breast cancer $(\mathrm{BC})$ is one of the leading malignant tumors threatening female health in China, with an increasing trend in incidence and mortality rates $(1,2)$. As a nongovernmental association, the Chinese Society of Clinical Oncology Breast Cancer (CSCO BC) is committed to promoting the standardization of $\mathrm{BC}$ diagnosis and treatment. In order to offer references for clinicians in $\mathrm{BC}$ diagnosis and treatment, CSCO BC arranged for a multidisciplinary panel of talented and dedicated breast surgeons, physicians, plastic surgeons, radiologists, pathologists, etc. With sustained efforts, CSCO BC developed the first version of its BC guideline in 2017, which has been the most distinguished and authoritative guideline of its kind in China.

The CSCO BC guidelines have been updated annually to reflect the rapidly increasing proportion of China's contribution to global BC research along with the significant changes in the accessibility of diagnosis and treatment resources. The updated 2020 CSCO BC guidelines were released on April 10, 2020. In the new version of the guidelines, the panel have made various of recommendations by incorporating content of the latest BC research from China and abroad, giving full consideration to the accessibility and affordability of diagnosis and treatment resources, and exploring more effective and reasonable stratifications of treatment options. This article mainly describes the three major Chinese aspects of the 2020 CSCO BC guideline updates.

\section{Integration of clinical experience and consensus from Chinese BC experts}

With regard to BC diagnosis and treatment, there are notable differences between China and other countries in clinicopathological characteristics, treatment patterns, drug accessibility, health insurance, and socioeconomic status. Due to epidemiological variations and lack of high-quality evidence concerning drug effectiveness and safety particularly in the Chinese population, some drugs have not been approved by the China Food and Drug Administration (CFDA, renamed to National Medical Products Administration or NMPA China in 2018) and thus have not yet been marketed. In addition, some therapies recommended by guidelines of other countries have not been covered by the National Reimbursement Drug List (NRDL), resulting in high out-of-pocket expenses for patients, and thus the application in these guidelines is cost prohibitive in China (3). These drugs are listed in the 2020 CSCO BC guidelines with low-level recommendation for their low accessibility and insufficient evidence. Compared to international guidelines which cannot reflect the diagnosis and treatment characteristics specific to Chinese BC patients, the 2020 CSCO BC guideline is more patientcentered and more tailored to Chinese patients' needs.

The 2020 CSCO BC guideline is not only concerned with benefitting patient, but also represents the consensus opinions of BC experts based on clinical experiences in China. Compared to other guidelines from abroad, CSCO BC guideline is more specific and applicable to 
the domestic treatment of BC. One notable strength of the CSCO BC guidelines is that molecular subtypes are considered when selecting patients eligible for neoadjuvant therapy. Traditionally, international $\mathrm{BC}$ guidelines, such as the National Comprehensive Cancer Network (NCCN) guidelines for $\mathrm{BC}$, have recommended using neoadjuvant therapy depending on tumor size, node status, and patient's preference. By contrast, as early as 2017, the CSCO BC panel developed different selection criteria of identifying appropriate candidates for neoadjuvant therapy with molecular subtypes included in the first version of the CSCO BC guideline. In the 2017 CSCO BC guideline, neoadjuvant therapy was indicated in patients with (I) large tumor size $(>5 \mathrm{~cm}$ ), (II) positive axillary nodes, (III) human epidermal growth factor receptor 2 (HER2)-positive type, (IV) triple-negative type, and (V) large primary tumor relative to breast size in patients who desire breast conservation. The selection criteria were also emphasized in the subsequent versions of CSCO BC guidelines. The panel decided that the indications of preoperative systemic therapy should no longer be determined by clinical stage alone. Rather, additional molecular subtypes should also be considered based on the current evidence that HER2-positive and triple-negative patients have a higher probability of achieving pathologic complete response (pCR) after neoadjuvant therapy (4). Similarly, the 2020 NCCN guidelines for BC were also updated to include HER2positive and triple-negative breast cancer (TNBC) tumors as indications for neoadjuvant therapy.

Another highlight of the 2020 CSCO BC guidelines is the separation of regimen recommendations for neoadjuvant and adjuvant therapies. In the NCCN guidelines for BC, both neoadjuvant and adjuvant therapies share the same regimens. However, the 2020 CSCO BC guideline presents different views on systemic therapy regimen recommendations. Although in general, any patient who is a candidate for adjuvant systemic therapy can be considered for neoadjuvant therapy, the CSCO panel determined that neoadjuvant and adjuvant regimens should be separated for the following reasons. First, neoadjuvant chemotherapy is administered before surgery with the short-term objective of shrinking the tumor to achieve pCR, while adjuvant therapy is delivered when the tumor is dissected with the goal of decreasing the possibility of recurrence. Consequently, neoadjuvant regimens should be aimed at rapidly achieving therapeutic benefits for patients. Second, because the neoadjuvant period provides a usable platform for the potential rapid assessment of drug efficacy and safety, there are more well-designed clinical trials involving neoadjuvant regimens with more new treatment options. Thus, there is more information for determining the optimal regimen in the neoadjuvant period than there is for the adjuvant period. Third, the neoadjuvant period can provide useful in-vivo information concerning the chemosensitivity of different regimens, which helps to guide subsequent drug selection (5). Therefore, recommendations for adjuvant therapy should be made based on response to neoadjuvant therapy.

It is also worth noting that one of the most significant updates in the 2020 CSCO BC guidelines is the optimization of neoadjuvant regimens for HER2-positive BC. The 2020 NCCN guidelines for BC list more options in the preferred regimens for neoadjuvant therapy, including both single HER2-blockade-based therapy and dual HER2-blockade-based therapy. By contrast, in the updated 2020 CSCO BC guidelines, only dual HER2targeted blockade with trastuzumab and pertuzumab, such as the docetaxel, carboplatin, trastuzumab, and pertuzumab (TCbHP) regimen, and the docetaxel, trastuzumab, pertuzumab (THP) regimen, is recommended as a level I recommendation for neoadjuvant therapy. It is generally recognized by the CSCO panel that all candidates for single-blockade therapy can also consider dual-blockade therapy in the neoadjuvant setting. Furthermore, through a comprehensive review of existing scientific evidence, the CSCO BC panel adjusted the recommendation levels for other treatment options regarding HER2positive $\mathrm{BC}$ neoadjuvant treatment. The doxorubicin and cyclophosphamide (AC)-THP regimen has been added to the level II recommendation with evidence category $2 \mathrm{~B}$; the $\mathrm{TCbH}$ regimen continues to be a level II recommendation, with its evidence category changed from $1 \mathrm{~A}$ to $2 \mathrm{~A}$. Scientifically and rationally designed clinical trials have also been added to the level II recommendation.

\section{Influential clinical trials published by Chinese experts}

Clinical trials conducted in China have contributed considerably to the development of this guideline. To help clinicians stay apprised of the new diagnostic approaches and treatment options from the latest literature and to help guide clinical decisions, the CSCO BC panel are committed to bringing attention to the latest domestic advances that have made an impact on global BC research. In the 2019 CSCO BC guidelines, 115 published articles were referenced, with Chinese literature accounting for to $24.3 \%$ 
(28/115) of the total; meanwhile, only $4.5 \%(18 / 699)$ of the references in the NCCN BC guidelines were Chinese based. Hence, the evidence provided in the CSCO BC guidelines more sufficiently reflect the characteristics of the Chinese population and healthcare policies. Here, we describe the four most influential clinical trials published by Chinese experts.

\section{The ACE trial}

In recent years, histone deacetylase inhibitor (HDAC) inhibitors have become a focal area of research due to their epigenetic regulation mechanism and their potential ability to overcome endocrine resistance. The ACE trial demonstrated that chidamide, an HDAC inhibitor, in combination with exemestane, can improve progressionfree survival (PFS) compared to exemestane alone (7.4 vs. 3.8 months; HR 0.75; 95\% CI, 0.58-0.98; $\mathrm{P}=0.033$ ) in patients with advanced $\mathrm{HR}$-positive $\mathrm{BC}$ progressed or relapsed after previous endocrine therapy (6). The results of the ACE study have led to the approval of chidamide by the CFDA for BC treatment. CSCO recommends combination therapy of chidamide and exemestane as the level I recommendation for patients with HR-positive or HER2negative advanced $\mathrm{BC}$ who fail tamoxifen or non-steroidal aromatase inhibitor (AI) therapy.

\section{The PEONY trial}

To investigate whether Asian patients with HER2-positive early or locally advanced BC benefit from the addition of pertuzumab to trastuzumab and docetaxel in the neoadjuvant setting compared with placebo, trastuzumab, and docetaxel, Shao and his colleagues conducted the PEONY trial. The researchers in this trial found that total pCR rates were higher with pertuzumab than with placebo (39.3\% vs. $21.8 \%$; 95\% CI, 6.9-28.0; $\mathrm{P}=0.001$ ). With respect to safety, the rates of common adverse events in this trial were in line with the known pertuzumab safety profile and comparable between groups, showing that the pertuzumab plus trastuzumab plus docetaxel regimen is well tolerated in the Asian population (7). Cited in the 2020 CSCO BC guidelines, the PEONY trial provides evidence for the benefit of the THP neoadjuvant regimen for HER2positive $\mathrm{BC}$ patients in China.

\section{The PHENIX and PHOEBE trials}

Based on the results of the PHENIX trial led by Jiang and the PHOEBE trial led by Binghe $\mathrm{Xu}$, the $2020 \mathrm{CSCO}$ $\mathrm{BC}$ guidelines recommend pyrotinib with capecitabine as the level I recommendation in the second-line therapy for HER2-positive advanced BC patients who fail trastuzumab therapy, and as the level III recommendation for HER2positive advanced BC patients who are naive or sensitive to trastuzumab; these recommendations are not included in the NCCN guidelines. The PHENIX trial showed that among women with HER2-positive metastatic BC, median PFS was improved with the combined pyrotinib and capecitabine regimen (11.1 months; 95\% CI, 9.66-16.53) compared to capecitabine alone (4.1 months; $95 \%$ CI, 2.79 4.17). Moreover, patients who progressed on capecitabine could also obtain benefit from pyrotinib treatment as a single agent, with an objective response rate of 38\% (95\% CI, 26.7-49.3\%) and a median PFS of 5.5 months (95\% CI, 4.07-6.9) (8). As part of the PHENIX trial, Binghe $\mathrm{Xu}$ led a phase II, randomized, multicenter, open-label study conducted in China (PHOEBE). This trial included Chinese patients with HER2-positive relapsed or metastatic BC previously treated with taxanes, anthracyclines, and/ or trastuzumab. These patients were randomly assigned $(1: 1)$ to receive pyrotinib or lapatinib in combination with capecitabine. The results showed that patients in the pyrotinib group had an approximately 11-month longer median PFS compared with patients in the lapatinib group (18.1 vs. 7.0 months; HR, 0.36; 95\% CI, 0.23-0.58; $\mathrm{P}<0.001)(9)$, indicating pyrotinib with capecitabine could be recommended as a treatment option for patients who are naive or sensitive to trastuzumab.

\section{Stratification of treatment recommendations based on drug accessibility and sensitivity}

The majority of clinical trials evaluate the efficacy of therapies in certain types of patients and are conducted in standardized conditions which reduce the interindividual variability. By contrast, in the real world, different tumor stages and a diversity in treatment history may alter the effectiveness and safety of drugs (10). In terms of offering recommendations about subsequent regimens, the CSCO $\mathrm{BC}$ panel thoroughly considered the influence of previous treatments. Compared to the NCCN guidelines in which recommended regimens are classified into "preferred regimens", "useful in certain circumstances", and "other recommended regimens", the updated 2020 version of CSCO BC guideline is more clinically based by stratifying treatment recommendations according to drug sensitivity 
and accessibility, instead of simply according to lines. Moreover, in the updated 2020 version of the CSCO $\mathrm{BC}$ guideline, the panel concentrated on exploring more effective ways of presenting recommended therapies, using tables and explanatory notes to cover stratification in treatment options for BC. The clear and detailed flowcharts designed by the CSCO panel $(11,12)$ are better presented and easier to follow compared to the illustrations, particularly the algorithms, in the NCCN guidelines.

In the 2019 CSCO BC guideline, the panel stratified HR-positive advanced BC into endocrine therapy (ET)naive, failure of tamoxifen (TAM) therapy, and failure of AI therapy. In this new version of the guideline, previous AI treatments are further divided into non-steroidal aromatase inhibitors (NSAIs) and steroidal aromatase inhibitors (SAIs), which is consistent with the enrollment groups of clinical trials. For ET-naive patients, the panel listed AI plus CDK4/6 inhibitors as a level I recommendation. For patients who previously failed TAM therapy, the level I recommendations include AI plus CDK4/6 inhibitors and fulvestrant plus CDK4/6 inhibitors. The panel also added AI plus HDAC inhibitors in level I recommendations based on the ACE trial. Single-agent endocrine therapy such as AI or fulvestrant alone has become a level II recommendation in the 2020 CSCO BC guideline instead of level I one as in the 2019 guideline. Single-agent endocrine therapy used to be the first choice for HRpositive advanced BC in China. However, in the updated CSCO BC guideline, combination therapies with CDK 4/6 inhibitors and endocrine therapy are preferred due to accessibility concerns and the high-quality evidence from several well-designed clinical trials (13-18). Although CDK 4/6 inhibitors have not yet been covered by the National Reimbursement Drug List, the panel believe that by raising recommendation levels, the charitable donations policy and government negotiation will make them more affordable for Chinese patients.

The 2020 CSCO BC guideline has been expanded and updated to include the "Adjuvant target therapy after neoadjuvant systemic therapy in HER2-positive breast cancer" section. To determine treatment options for adjuvant therapy, patients are stratified according to types of neoadjuvant therapy received and the attainment of pCR. In the case of dual HER2-blockade (TCbHP or THP) received as neoadjuvant therapy, a trastuzumab and pertuzumab (HP) regimen should be maintained as an adjuvant regimen if pCR is achieved. For non-pCR patients, trastuzumab emtansine (T-DM1) is a level I recommendation, while HP is a level II recommendation. For patients who receive single HER2-blockade (TH-based regimen) as neoadjuvant therapy, if $\mathrm{pCR}$ is achieved, then continuing trastuzumab is a level I recommendation, with the HP regimen listed as level II recommendation. If pCR is not achieved, HP regimen and T-DM1 regimen are the level I recommendation. Because there is no direct evidence regarding the use of the adjuvant HP regimen for non-pCR patients after previous neoadjuvant trastuzumab treatment, the T-DM1 regimen is listed in a higher evidence category (1B) than the HP regimen (2A). However, since T-DM1 is not covered by health insurance, the CSCO panel prefer $\mathrm{HP}$ regimen for its superior accessibility.

As a trastuzumab-based scheme is recommended as the first-line therapy by almost every set of guidelines, HER2positive metastatic $\mathrm{BC}$ is stratified into trastuzumab-sensitive and trastuzumab-resistant categories in the updated CSCO $\mathrm{BC}$ guidelines, which are more appropriate and more clearly defined than the "first line" or "second line" for routine clinical practice. For trastuzumab-sensitive patients, THP regimen and trastuzumab combined with chemotherapy are recommended. Meanwhile, for trastuzumab-resistant patients, TKI-containing regimens and T-DM1 are listed as recommendations. The panel believe that these changes will bring more convenience to clinical decision-making

\section{Conclusions}

The CSCO BC guidelines have both Chinese roots and global reach. On the path of guideline implementation, significant achievements have been made step by step: from referring to and translating NCCN guidelines, to participation in international consensus meetings, to cooperation in clinical trials, to organization of real-world studies, and to receiving increasing global recognition. With the joint effort of clinicians, researchers, statisticians, pharmaceutical enterprises, and public health authorities, the CSCO BC panel will continue to translate the latest research into clinical practice and offer recommendations based on drug accessibility. More and greater advances are expected in the further optimization of the CSCO BC guidelines. While much work still needs to be done, we believe these guidelines will continue to make an important contribution towards promoting standardized treatment of $\mathrm{BC}$ and towards making cure a feasible goal for Chinese patients in the near future. 


\section{Acknowledgments}

Funding: None.

\section{Footnote}

Provenance and Peer Review: This article was commissioned by the Editorial Office, Translational Breast Cancer Research. The article has undergone external peer review.

Conflicts of Interest: All authors have completed the ICMJE uniform disclosure form (available at http://dx.doi. org/10.21037/tbcr-20-50). ZJ serves as an unpaid Editorin-Chief of Translational Breast Cancer Research. The other authors have no conflicts of interest to declare.

Ethical Statement: The authors are accountable for all aspects of the work in ensuring that questions related to the accuracy or integrity of any part of the work are appropriately investigated and resolved.

Open Access Statement: This is an Open Access article distributed in accordance with the Creative Commons Attribution-NonCommercial-NoDerivs 4.0 International License (CC BY-NC-ND 4.0), which permits the noncommercial replication and distribution of the article with the strict proviso that no changes or edits are made and the original work is properly cited (including links to both the formal publication through the relevant DOI and the license). See: https://creativecommons.org/licenses/by-nc-nd/4.0/.

\section{References}

1. Chen W, Zheng R, Baade PD, et al. Cancer statistics in China, 2015. CA Cancer J Clin 2016;66:115-32.

2. Zheng RS, Sun KX, Zhang SW, et al. Report of cancer epidemiology in China, 2015. Zhonghua Zhong Liu Za Zhi 2019;41:19-28.

3. Fan L, Strasser-Weippl K, Li JJ, et al. Breast cancer in China. Lancet Oncol 2014;15:e279-89.

4. Cortazar P, Zhang L, Untch M, et al. Pathological complete response and long-term clinical benefit in breast cancer: the CTNeoBC pooled analysis. Lancet 2014;384:164-72.

5. Colomer R, Saura C, Sanchez-Rovira P, et al. Neoadjuvant Management of Early Breast Cancer: A Clinical and Investigational Position Statement. Oncologist 2019;24:603-11.
6. Jiang Z, Li W, Hu X, et al. Tucidinostat plus exemestane for postmenopausal patients with advanced, hormone receptor-positive breast cancer (ACE): a randomised, double-blind, placebo-controlled, phase 3 trial. Lancet Oncol 2019;20:806-15.

7. Shao Z, Pang D, Yang H, et al. Efficacy, Safety, and Tolerability of Pertuzumab, Trastuzumab, and Docetaxel for Patients With Early or Locally Advanced ERBB2Positive Breast Cancer in Asia: The PEONY Phase 3 Randomized Clinical Trial. JAMA Oncol 2020;6:e193692.

8. Jiang Z. Pyrotinib combined with capecitabine in women with HER2 + metastatic breast cancer previously treated with trastuzumab and taxanes: A randomized phase III study. Chicago: Proceedings of the ASCO Annual Meeting, May 31-June 4, 2019.

9. Ma F, Ouyang Q, Li W, et al. Pyrotinib or Lapatinib Combined With Capecitabine in HER2-Positive Metastatic Breast Cancer With Prior Taxanes, Anthracyclines, and/or Trastuzumab: A Randomized, Phase II Study. J Clin Oncol 2019;37:2610-9.

10. Pablos-Méndez A, Barr G, Shea S. Run-in periods in randomized trials. Implications for the application of results in clinical practice. JAMA 1998;279:222-5.

11. Fengrui X, Zefei J. CSCO BC guideline: updates for hormone receptor-positive breast cancer in 2020. Transl Breast Cancer Res 2020;1:3.

12. Jianbin L, Zefei J. CSCO BC guideline: updates for HER2 positive breast cancer in 2020. Transl Breast Cancer Res 2020;1:4.

13. Finn RS, Crown JP, Lang I, et al. The cyclin-dependent kinase 4/6 inhibitor palbociclib in combination with letrozole versus letrozole alone as first-line treatment of oestrogen receptor-positive, HER2-negative, advanced breast cancer (PALOMA-1/TRIO-18): a randomised phase 2 study. Lancet Oncol 2015;16:25-35.

14. Finn RS, Martin M, Rugo HS, et al. Palbociclib and Letrozole in Advanced Breast Cancer. N Engl J Med 2016;375:1925-36.

15. Cristofanilli M, Turner NC, Bondarenko I, et al. Fulvestrant plus palbociclib versus fulvestrant plus placebo for treatment of hormone-receptor-positive, HER2negative metastatic breast cancer that progressed on previous endocrine therapy (PALOMA-3): final analysis of the multicentre, double-blind, phase 3 randomised controlled trial. Lancet Oncol 2016;17:425-39.

16. Turner NC, Slamon DJ, Ro J, et al. Overall Survival with Palbociclib and Fulvestrant in Advanced Breast Cancer. N Engl J Med 2018;379:1926-36. 
17. Hortobagyi GN, Stemmer SM, Burris HA, et al. Updated results from MONALEESA-2, a phase III trial of firstline ribociclib plus letrozole versus placebo plus letrozole in hormone receptor-positive, HER2-negative advanced breast cancer. Ann Oncol 2018;29:1541-7.

18. Sledge GW Jr, Toi M, Neven P, Sohn J, et al. MONARCH

doi: $10.21037 /$ tbcr-20-50

Cite this article as: Shen B, Wang K, Jiang Z. 2020 Chinese

Society of Clinical Oncology Breast Cancer guideline updates.

Transl Breast Cancer Res 2020;1:25.
2: Abemaciclib in Combination With Fulvestrant in Women With HR+/HER2- Advanced Breast Cancer Who Had Progressed While Receiving Endocrine Therapy. J Clin Oncol 2017;35:2875-84.

(English Language Editor: J. Gray) 